\title{
Bastadins and bastaranes-enhanced precatalyst preparation stabilization and initiation (EPPSI) nano molecules
}

\author{
Alireza Heidari* \\ Faculty of Chemistry, California South University, 14731 Comet St. Irvine, CA 92604, USA
}

In the current editorial, we study Bastadins and BastaranesEnhanced Precatalyst Preparation Stabilization and Initiation (EPPSI) Nano molecules incorporation into the Nano Polymeric Matrix (NPM) by immersion of the Nano Polymeric Modified Electrode (NPME) as molecular enzymes and drug targets for human cancer cells, tissues and tumors treatment under synchrotron and synchrocyclotron radiations (Figure 1). Bastadins are molecules isolated from the marine sponge Ianthella basta. They possess antibacterial, cytotoxic and antiinflammatory properties. Bastaranes and isobastaranes are derivatives of these. To keep track of them all, the bastadins are numbered, bastadin-1, bastadin-2, etc. The molecule shown in the diagram is bastadin-5, which is one of the few commercially available ones. In this regard, the development of Chemical Modified Electrodes (CEMs) is at present an area of great interest. CEMs can be divided broadly into two main categories; namely, surface modified and bulk modified electrodes. Methods of surface modification include adsorption, covalent bonding, attachment of polymer Nano films, etc. Polymer Nano film coated electrodes can be differentiated from other modification methods such as adsorption and covalent bonding in that they usually involve multilayer as opposed to monolayer frequently encountered for the latter methods. The thicker Nano films imply more active sites which
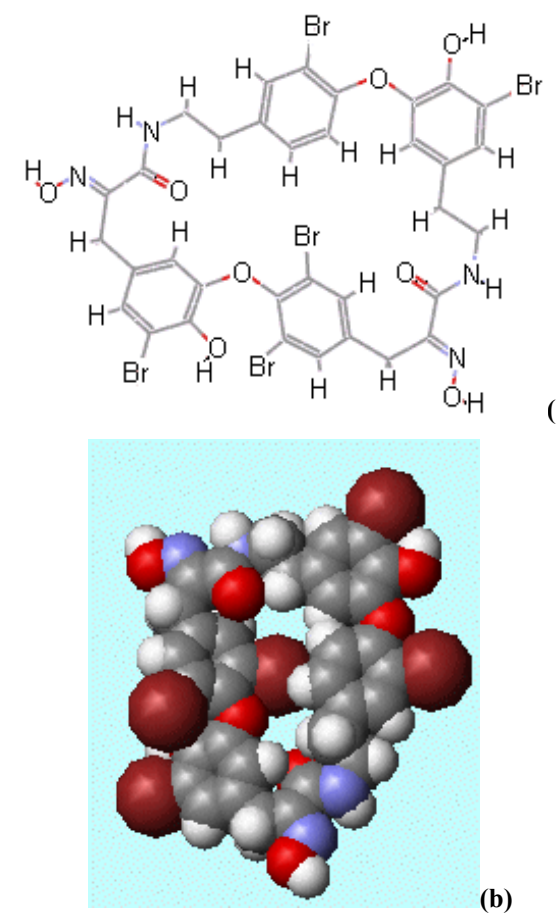

(b)

Figure 1. Molecular structure of (a) bastadins and (b) bastaranes nano molecules lead to larger analytical signals. This advantage coupled with other, their versatility and wide applicability, makes polymer Nano film modified electrodes particularly suitable for analytical applications [1-27].

Electrochemical polymerization offers the advantage of reproducible deposition in terms of Nano film thickness and loading, making the immobilization procedure of a metal-based electrocatalyst very simple and reliable for Bastadins and Bastaranes-Enhanced Precatalyst Preparation Stabilization and Initiation (EPPSI) Nano molecules-encapsulating Carbon nanotubes incorporation into the Nano Polymeric Matrix (NPM) by immersion of the Nano Polymeric Modified Electrode (NPME) as molecular enzymes and drug targets for human cancer cells, tissues and tumors treatment under synchrotron and synchrocyclotron radiations. Also, it must be notice that the nature of working electrode substrate in electropreparation of polymeric Nano film is very important, because properties of polymeric Nano films depend on the working electrode anti-cancer Nano materials. The ease and fast preparation and of obtaining a new reproducible surface, the low residual current, porous surface and low cost of Multi-Walled Carbon Nanotubes (MWCNTs) paste are some advantages of Carbon Paste Electrode (CPE) over all other solid electrodes [28-92].

On the other hand, it has been shown that, macrocyclic complexes of Bastadins and Bastaranes-Enhanced Precatalyst Preparation Stabilization and Initiation (EPPSI) Nano molecules-encapsulating Carbon nanotubes are interest as modifying agents because in basic media Bastadins and Bastaranes-Enhanced Precatalyst Preparation Stabilization and Initiation (EPPSI) Nano molecules-encapsulating Carbon nanotubes redox centers show high catalytic activity towards the oxidation of small organic anti-cancer Nano compounds. The highvalence species of Bastadins and Bastaranes-Enhanced Precatalyst Preparation Stabilization and Initiation (EPPSI) Nano moleculesencapsulating Carbon nanotubes seem to act as strong oxidizing agents for low-electroactivity organic substrates. 1,2-Dioxetane $(1,2-$ Dioxacyclobutane), 1,3-Dioxetane (1,3-Dioxacyclobutane), DMDM Hydantoin and Sulphobe as the anti-cancer organic intermediate products of methanol oxidation as well as formic acid, is important to investigate its electrochemical oxidation behavior in Bastadins and Bastaranes-Enhanced Precatalyst Preparation Stabilization and Initiation (EPPSI) Nano molecules-encapsulating Carbon nanotubes incorporation into the Nano Polymeric Matrix (NPM) by immersion of

${ }^{*}$ Correspondence to: Alireza Heidari, Faculty of Chemistry, California South University, 14731 Comet St. Irvine, CA 92604, USA, E-mail: Scholar.Researcher. Scientist@gmail.com; Alireza.Heidari@calsu.us

Received: July 31, 2018; Accepted: August 13, 2018; Published: August 15, 2018 
the Nano Polymeric Modified Electrode (NPME) as molecular enzymes and drug targets for human cancer cells, tissues and tumors treatment under synchrotron and synchrocyclotron radiations [93-110].

In this editorial, we decided to combine the above-mentioned advantageous features for the aim of Bastadins and BastaranesEnhanced Precatalyst Preparation Stabilization and Initiation (EPPSI) Nano molecules-encapsulating Carbon nanotubes incorporation into the Nano Polymeric Matrix (NPM) by immersion of the Nano Polymeric Modified Electrode (NPME) as molecular enzymes and drug targets for human cancer cells, tissues and tumors treatment under synchrotron and synchrocyclotron radiations. Furthermore, in this editorial, we prepared poly Nano films by electropolymerization at the surface of Multi-Walled Carbon Nanotubes (MWCNTs) paste electrode. Then, Bastadins and Bastaranes-Enhanced Precatalyst Preparation Stabilization and Initiation (EPPSI) Nano molecules-encapsulating Carbon nanotubes were incorporated into the Nano Polymeric Matrix (NPM) by immersion of the Nano Polymeric Modified Electrode (NPME) in a solution. The modifier layer of Bastadins and BastaranesEnhanced Precatalyst Preparation Stabilization and Initiation (EPPSI) Nano molecules-encapsulating Carbon nanotubes at the electrode surface acts as a Nano catalyst for the treatment of human cancer cells, tissues and tumors under synchrotron and synchrocyclotron radiations. Suitability of this Bastadins and Bastaranes-Enhanced Precatalyst Preparation Stabilization and Initiation (EPPSI) Nano molecules-encapsulating Carbon nanotubes-modified polymeric Multi-Walled Carbon Nanotubes (MWCNTs) paste electrode toward the electrocatalytic treatment of human cancer cells, tissues and tumors under synchrotron and synchrocyclotron radiations in alkaline medium at ambient temperature was investigated [111-182].

\section{References}

1. Alireza Heidari, Christopher Brown (2015) Study of Composition and Morphology of Cadmium Oxide (CdO) Nanoparticles for Eliminating Cancer Cells. Journal of Nanomedicine Research, Volume 2, Issue 5, 20 Pages.

2. Alireza Heidari, Christopher Brown (2015) Study of Surface Morphological, Phytochemical and Structural Characteristics of Rhodium (III) Oxide (Rh2O3) Nanoparticles. International Journal of Pharmacology, Phytochemistry and Ethnomedicine, Volume 1, Pages 15-19.

3. Alireza Heidari (2016) An Experimental Biospectroscopic Study on Seminal Plasma in Determination of Semen Quality for Evaluation of Male Infertility. Int J Adv Technol 7: e007.

4. Alireza Heidari (2016) Extraction and Preconcentration of N-Tolyl-SulfonylPhosphoramid-Saeure-Dichlorid as an Anti-Cancer Drug from Plants: A Pharmacognosy Study. J Pharmacogn Nat Prod 2: e103.

5. Alireza Heidari (2016) A Thermodynamic Study on Hydration and Dehydration of DNA and RNA-Amphiphile Complexes. J Bioeng Biomed Sci S: 006.

6. Alireza Heidari (2016) Computational Studies on Molecular Structures and Carbonyl and Ketene Groups' Effects of Singlet and Triplet Energies of Azidoketene $\mathrm{O}=\mathrm{C}=\mathrm{CH}-$ $\mathrm{NNN}$ and Isocyanatoketene $\mathrm{O}=\mathrm{C}=\mathrm{CH}-\mathrm{N}=\mathrm{C}=\mathrm{O}$. J Appl Computat Math 5: e142.

7. Alireza Heidari (2016) Study of Irradiations to Enhance the Induces the Dissociation of Hydrogen Bonds between Peptide Chains and Transition from Helix Structure to Random Coil Structure Using ATR-FTIR, Raman and 1HNMR Spectroscopies. J Biomol Res Ther 5: e146.

8. Alireza Heidari (2016) Future Prospects of Point Fluorescence Spectroscopy, Fluorescence Imaging and Fluorescence Endoscopy in Photodynamic Therapy (PDT) for Cancer Cells. J Bioanal Biomed 8: e135.

9. Alireza Heidari (2016) A Bio-Spectroscopic Study of DNA Density and Color Role as Determining Factor for Absorbed Irradiation in Cancer Cells. Adv Cancer Prev 1: e102.

10. Alireza Heidari (2016) Manufacturing Process of Solar Cells Using Cadmium Oxide (CdO) and Rhodium (III) Oxide (Rh2O3) Nanoparticles. J Biotechnol Biomater 6: e125.
11. Alireza Heidari (2016) A Novel Experimental and Computational Approach to Photobiosimulation of Telomeric DNA/RNA: A Biospectroscopic and Photobiological Study. J Res Development 4: 144.

12. Alireza Heidari (2016) Biochemical and Pharmacodynamical Study of Microporous Molecularly Imprinted Polymer Selective for Vancomycin, Teicoplanin, Oritavancin, Telavancin and Dalbavancin Binding. Biochem Physiol 5: e146.

13. Alireza Heidari (2016) Anti-Cancer Effect of UV Irradiation at Presence of Cadmium Oxide (CdO) Nanoparticles on DNA of Cancer Cells: A Photodynamic Therapy Study. Arch Cancer Res. 4: 1.

14. Alireza Heidari (2016) Biospectroscopic Study on Multi-Component Reactions (MCRs) in Two A-Type and B-Type Conformations of Nucleic Acids to Determine Ligand Binding Modes, Binding Constant and Stability of Nucleic Acids in Cadmium Oxide (CdO) Nanoparticles-Nucleic Acids Complexes as Anti-Cancer Drugs. Arch Cancer Res. 4: 2 .

15. Alireza Heidari (2016) Simulation of Temperature Distribution of DNA/RNA of Human Cancer Cells Using Time-Dependent Bio-Heat Equation and Nd: YAG Lasers. Arch Cancer Res. 4: 2.

16. Alireza Heidari (2016) Quantitative Structure-Activity Relationship (QSAR) Approximation for Cadmium Oxide (CdO) and Rhodium (III) Oxide (Rh2O3) Nanoparticles as Anti-Cancer Drugs for the Catalytic Formation of Proviral DNA from Viral RNA Using Multiple Linear and Non-Linear Correlation Approach. Ann Clin Lab Res. 4: 1.

17. Alireza Heidari (2016) Biomedical Study of Cancer Cells DNA Therapy Using Laser Irradiations at Presence of Intelligent Nanoparticles. J Biomedical Sci. 5: 2.

18. Alireza Heidari (2016) Measurement the Amount of Vitamin D2 (Ergocalciferol), Vitamin D3 (Cholecalciferol) and Absorbable Calcium (Ca2+), Iron (II) (Fe2+), Magnesium (Mg2+), Phosphate (PO4-) and Zinc (Zn2+) in Apricot Using HighPerformance Liquid Chromatography (HPLC) and Spectroscopic Techniques. J Biom Biostat 7: 292.

19. Alireza Heidari (2016) Spectroscopy and Quantum Mechanics of the Helium Dimer $(\mathrm{He} 2+)$, Neon Dimer (Ne2+), Argon Dimer (Ar2+), Krypton Dimer (Kr2+), Xenon Dimer $(\mathrm{Xe} 2+)$, Radon Dimer(Rn2+) and Ununoctium Dimer (Uuo2+) Molecular Cations. Chem Sci J 7: e112.

20. Alireza Heidari (2016) Human Toxicity Photodynamic Therapy Studies on DNA/RNA Complexes as a Promising New Sensitizer for the Treatment of Malignant Tumors Using Bio-Spectroscopic Techniques. J Drug Metab Toxicol 7: e129.

21. Alireza Heidari (2016) Novel and Stable Modifications of Intelligent Cadmium Oxide (CdO) Nanoparticles as Anti-Cancer Drug in Formation of Nucleic Acids Complexes for Human Cancer Cells' Treatment. Biochem Pharmacol (Los Angel) 5: 207.

22. Alireza Heidari (2016) A Combined Computational and QM/MM Molecular Dynamics Study on Boron Nitride Nanotubes (BNNTs), Amorphous Boron Nitride Nanotubes (aBNNTs) and Hexagonal Boron Nitride Nanotubes (h-BNNTs) as Hydrogen Storage. Struct Chem Crystallogr Commun 2: 1 .

23. Alireza Heidari (2016) Pharmaceutical and Analytical Chemistry Study of Cadmium Oxide (CdO) Nanoparticles Synthesis Methods and Properties as Anti-Cancer Drug and its Effect on Human Cancer Cells. Pharm Anal Chem Open Access 2: 113.

24. Alireza Heidari (2016) A Chemotherapeutic and Biospectroscopic Investigation of the Interaction of Double-Standard DNA/RNA-Binding Molecules with Cadmium Oxide (CdO) and Rhodium (III) Oxide (Rh2O3) Nanoparticles as Anti-Cancer Drugs for Cancer Cells' Treatment. Chemo Open Access 5: e129.

25. Alireza Heidari (2016) Pharmacokinetics and Experimental Therapeutic Study of DNA and Other Biomolecules Using Lasers: Advantages and Applications. J Pharmacokinet Exp Ther 1: e005.

26. Alireza Heidari (2016) Determination of Ratio and Stability Constant of DNA/RNA in Human Cancer Cells and Cadmium Oxide (CdO) Nanoparticles Complexes Using Analytical Electrochemical and Spectroscopic Techniques. Insights Anal Electrochem 2: 1 .

27. Alireza Heidari (2016) Discriminate between Antibacterial and Non-Antibacterial Drugs Artificial Neutral Networks of a Multilayer Perceptron (MLP) Type Using a Set of Topological Descriptors. J Heavy Met Toxicity Dis. 1: 2.

28. Alireza Heidari (2016) Combined Theoretical and Computational Study of the Belousov-Zhabotinsky Chaotic Reaction and Curtius Rearrangement for Synthesis of Mechlorethamine, Cisplatin, Streptozotocin, Cyclophosphamide, Melphalan, Busulphan and BCNU as Anti-Cancer Drugs. Insights Med Phys. 1: 2.

29. Alireza Heidari (2016) A Translational Biomedical Approach to Structural Arrangement of Amino Acids' Complexes: A Combined Theoretical and Computational Study. Transl Biomed. 7: 2. 
30. Alireza Heidari (2016) Ab Initio and Density Functional Theory (DFT) Studies of Dynamic NMR Shielding Tensors and Vibrational Frequencies of DNA/RNA and Cadmium Oxide (CdO) Nanoparticles Complexes in Human Cancer Cells. J Nanomedine Biotherapeutic Discov 6: e144.

31. Alireza Heidari (2016) Molecular Dynamics and Monte-Carlo Simulations for Replacement Sugars in Insulin Resistance, Obesity, LDL Cholesterol, Triglycerides, Metabolic Syndrome, Type 2 Diabetes and Cardiovascular Disease: A Glycobiological Study. J Glycobiol 5: e111.

32. Alireza Heidari (2016) Synthesis and Study of 5-[(Phenylsulfonyl)Amino]-1,3,4 Thiadiazole-2-Sulfonamide as Potential Anti-Pertussis Drug Using Chromatography and Spectroscopy Techniques. Transl Med (Sunnyvale) 6: e138.

33. Alireza Heidari (2016) Nitrogen, Oxygen, Phosphorus and Sulphur Heterocyclic AntiCancer Nano Drugs Separation in the Supercritical Fluid of Ozone (O3) Using SoaveRedlich-Kwong (SRK) and Pang-Robinson (PR) Equations. Electronic J Biol 12: 4.

34. Alireza Heidari (2016) An Analytical and Computational Infrared Spectroscopic Review of Vibrational Modes in Nucleic Acids. Austin J Anal Pharm Chem. 3 (1): 1058.

35. Alireza Heidari, Christopher Brown (2016) Phase, Composition and Morphology Study and Analysis of Os-Pd/HfC Nanocomposites. Nano Res Appl. 2: 1.

36. Alireza Heidari, Christopher Brown (2016) Vibrational Spectroscopic Study of Intensities and Shifts of Symmetric Vibration Modes of Ozone Diluted by Cumene. International Journal of Advanced Chemistry, 4 (1) 5-9.

37. Alireza Heidari (2016) Study of the Role of Anti-Cancer Molecules with Different Sizes for Decreasing Corresponding Bulk Tumor Multiple Organs or Tissues. Arch Can Res. 4: 2 .

38. Alireza Heidari (2016) Genomics and Proteomics Studies of Zolpidem, Necopidem, Alpidem, Saripidem, Miroprofen, Zolimidine, Olprinone and Abafungin as AntiTumor, Peptide Antibiotics, Antiviral and Central Nervous System (CNS) Drugs. J Data Mining Genomics \& Proteomics 7: e125.

39. Alireza Heidari (2016) Pharmacogenomics and Pharmacoproteomics Studies of Phosphodiesterase-5 (PDE5) Inhibitors and Paclitaxel Albumin-Stabilize Nanoparticles as Sandwiched Anti-Cancer Nano Drugs between Two DNA/RNA Molecules of Human Cancer Cells. J Pharmacogenomics Pharmacoproteomics 7: e153.

40. Alireza Heidari (2016) Biotranslational Medical and Biospectroscopic Studies of Cadmium Oxide (CdO) Nanoparticles-DNA/RNA Straight and Cycle Chain Complexes as Potent Anti-Viral, Anti-Tumor and Anti-Microbial Drugs: A Clinical Approach. Transl Biomed. 7: 2.

41. Alireza Heidari (2016) A Comparative Study on Simultaneous Determination and Separation of Adsorbed Cadmium Oxide (CdO) Nanoparticles on DNA/RNA of Human Cancer Cells Using Biospectroscopic Techniques and Dielectrophoresis (DEP) Method. Arch Can Res. 4: 2

42. Alireza Heidari (2016) Cheminformatics and System Chemistry of Cisplatin, Carboplatin, Nedaplatin, Oxaliplatin, Heptaplatin and Lobaplatin as Anti-Cancer Nano Drugs: A Combined Computational and Experimental Study. J Inform Data Min 1: 3.

43. Alireza Heidari (2016) Linear and Non-Linear Quantitative Structure-Anti-CancerActivity Relationship (QSACAR) Study of Hydrous Ruthenium (IV) Oxide (RuO2) Nanoparticles as Non-Nucleoside Reverse Transcriptase Inhibitors (NNRTIs) and Anti-Cancer Nano Drugs. J Integr Oncol 5: e110.

44. Alireza Heidari (2016) Synthesis, Characterization and Biospectroscopic Studies of Cadmium Oxide (CdO) Nanoparticles-Nucleic Acids Complexes Absence of Soluble Polymer as a Protective Agent Using Nucleic Acids Condensation and Solution Reduction Method. J Nanosci Curr Res 1: e101.

45. Alireza Heidari (2016) Coplanarity and Collinearity of 4'-Dinonyl-2,2'-Bithiazole in One Domain of Bleomycin and Pingyangmycin to be Responsible for Binding of Cadmium Oxide (CdO) Nanoparticles to DNA/RNA Bidentate Ligands as Anti-Tumor Nano Drug. Int J Drug Dev \& Res 8: 007-008.

46. Alireza Heidari (2016) A Pharmacovigilance Study on Linear and Non-Linear Quantitative Structure (Chromatographic) Retention Relationships (QSRR) Models for the Prediction of Retention Time of Anti-Cancer Nano Drugs under Synchrotron Radiations. J Pharmacovigil 4: e161.

47. Alireza Heidari (2016) Nanotechnology in Preparation of Semipermeable Polymers. J Adv Chem Eng 6: 157.

48. Alireza Heidari (2016) A Gastrointestinal Study on Linear and Non-Linear Quantitative Structure (Chromatographic) Retention Relationships (QSRR) Models for Analysis 5Aminosalicylates Nano Particles as Digestive System Nano Drugs under Synchrotron Radiations. J Gastrointest Dig Syst 6: e119.
49. Alireza Heidari (2016) DNA/RNA Fragmentation and Cytolysis in Human Cancer Cells Treated with Diphthamide Nano Particles Derivatives. Biomedical Data Mining 5: e102.

50. Alireza Heidari (2016) A Successful Strategy for the Prediction of Solubility in the Construction of Quantitative Structure-Activity Relationship (QSAR) and Quantitative Structure-Property Relationship (QSPR) under Synchrotron Radiations Using Genetic Function Approximation (GFA) Algorithm. J Mol Biol Biotechnol 1: 1.

51. Alireza Heidari (2016) Computational Study on Molecular Structures of C20, C60 C240, C540, C960, C2160 and C3840 Fullerene Nano Molecules under Synchrotron Radiations Using Fuzzy Logic. J Material Sci Eng 5: 282.

52. Alireza Heidari (2016) Graph Theoretical Analysis of Zigzag Polyhexamethylene Biguanide, Polyhexamethylene Adipamide, Polyhexamethylene Biguanide Gauze and Polyhexamethylene Biguanide Hydrochloride (PHMB) Boron Nitride Nanotubes (BNNTs), Amorphous Boron Nitride Nanotubes (a-BNNTs) and Hexagonal Boron Nitride Nanotubes (h-BNNTs). J Appl Computat Math 5: e143.

53. Alireza Heidari (2016) The Impact of High Resolution Imaging on Diagnosis. Int J Clin Med Imaging 3: 1000e101.

54. Alireza Heidari (2016) A Comparative Study of Conformational Behavior of Isotretinoin (13-Cis Retinoic Acid) and Tretinoin (All-Trans Retinoic Acid (ATRA)) Nano Particles as Anti-Cancer Nano Drugs under Synchrotron Radiations Using Hartree-Fock (HF) and Density Functional Theory (DFT) Methods. Insights in Biomed $1: 2$.

55. Alireza Heidari (2016) Advances in Logic, Operations and Computational Mathematics. J Appl Computat Math 5: 5.

56. Alireza Heidari (2016) Mathematical Equations in Predicting Physical Behavior. J Appl Computat Math 5: 5.

57. Alireza Heidari (2016) Chemotherapy a Last Resort for Cancer Treatment. Chemo Open Access 5: 4.

58. Alireza Heidari (2016) Separation and Pre-Concentration of Metal Cations-DNA/RNA Chelates Using Molecular Beam Mass Spectrometry with Tunable Vacuum Ultraviolet (VUV) Synchrotron Radiation and Various Analytical Methods. Mass Spectrom Purif Tech 2: e101.

59. Alireza Heidari (2016) Yoctosecond Quantitative Structure-Activity Relationship (QSAR) and Quantitative Structure-Property Relationship (QSPR) under Synchrotron Radiations Studies for Prediction of Solubility of Anti-Cancer Nano Drugs in Aqueous Solutions Using Genetic Function Approximation (GFA) Algorithm. Insight Pharm Res. 1: 1.

60. Alireza Heidari (2016) Cancer Risk Prediction and Assessment in Human Cells under Synchrotron Radiations Using Quantitative Structure Activity Relationship (QSAR) and Quantitative Structure Properties Relationship (QSPR) Studies. Int J Clin Med Imaging 3: 516 .

61. Alireza Heidari (2016) A Novel Approach to Biology. Electronic J Biol 12: 4.

62. Alireza Heidari (2016) Innovative Biomedical Equipment's for Diagnosis and Treatment. J Bioengineer \& Biomedical Sci 6: 2

63. Alireza Heidari (2016) Integrating Precision Cancer Medicine into Healthcare, Medicare Reimbursement Changes and the Practice of Oncology: Trends in Oncology Medicine and Practices. J Oncol Med \& Pract 1: 2.

64. Alireza Heidari (2016) Promoting Convergence in Biomedical and Biomaterials Sciences and Silk Proteins for Biomedical and Biomaterials Applications: An Introduction to Materials in Medicine and Bioengineering Perspectives. J Bioengineer \& Biomedical Sci 6: 3

65. Alireza Heidari (2017) X-Ray Fluorescence and X-Ray Diffraction Analysis on Discrete Element Modeling of Nano Powder Metallurgy Processes in Optimal Container Design. J Powder Metall Min 6: 1.

66. Alireza Heidari (2017) Biomolecular Spectroscopy and Dynamics of Nano-Sized Molecules and Clusters as Cross-Linking-Induced Anti-Cancer and ImmuneOncology Nano Drugs Delivery in DNA/RNA of Human Cancer Cells' Membranes under Synchrotron Radiations: A Payload-Based Perspective. Arch Chem Res. 1: 2.

67. Alireza Heidari (2017) Deficiencies in Repair of Double-Standard DNA/RNABinding Molecules Identified in Many Types of Solid and Liquid Tumors Oncology in Human Body for Advancing Cancer Immunotherapy Using Computer Simulations and Data Analysis. J Appl Bioinforma Comput Biol, 6: 1.

68. Alireza Heidari (2017) Electronic Coupling among the Five Nanomolecules Shut Down Quantum Tunneling in the Presence and Absence of an Applied Magnetic Field for Indication of the Dimer or other Provide Different Influences on the Magnetic Behavior of Single Molecular Magnets (SMMs) as Qubits for Quantum Computing. Glob J Res Rev. 4: 2. 
69. Alireza Heidari (2017) Polymorphism in Nano-Sized Graphene Ligand-Induced Transformation of Au38-xAgx/xCux(SPh-tBu)24 to Au36-xAgx/xCux(SPh-tBu)24 $(\mathrm{x}=1-12)$ Nanomolecules for Synthesis of Au144-xAgx/xCux[(SR)60, (SC4)60, (SC6)60, (SC12)60, (PET)60, (p-MBA)60, (F)60, (Cl)60, (Br)60, (I)60, (At)60, (Uus)60 and (SC6H13)60] Nano Clusters as Anti-Cancer Nano Drugs. J Nanomater Mol Nanotechnol, 6: 3 .

70. Alireza Heidari (2017) Biomedical Resource Oncology and Data Mining to Enable Resource Discovery in Medical, Medicinal, Clinical, Pharmaceutical, Chemical and Translational Research and Their Applications in Cancer Research. Int J Biomed Data Min 6: e103.

71. Alireza Heidari (2017) Study of Synthesis, Pharmacokinetics, Pharmacodynamics, Dosing, Stability, Safety and Efficacy of Olympiadane Nanomolecules as Agent for Cancer Enzymotherapy, Immunotherapy, Chemotherapy, Radiotherapy, Hormone Therapy and Targeted Therapy under Synchrotorn Radiation. J Dev Drugs 6: e154.

72. Alireza Heidari (2017) A Novel Approach to Future Horizon of Top Seven Biomedical Research Topics to Watch in 2017: Alzheimer's, Ebola, Hypersomnia, Human Immunodeficiency Virus (HIV), Tuberculosis (TB), Microbiome/Antibiotic Resistance and Endovascular Stroke. J Bioengineer \& Biomedical Sci 7: e127.

73. Alireza Heidari (2017) Opinion on Computational Fluid Dynamics (CFD) Technique Fluid Mech Open Acc 4: 157

74. Alireza Heidari (2017) Concurrent Diagnosis of Oncology Influence Outcomes in Emergency General Surgery for Colorectal Cancer and Multiple Sclerosis (MS) Treatment Using Magnetic Resonance Imaging (MRI) and Au329(SR)84, Au329xAgx(SR)84, Au144(SR)60, Au68(SR)36, Au30(SR)18, Au102(SPh)44, Au38(SPh)24, Au38(SC2H4Ph)24, Au21S(SAdm)15, Au36(pMBA)24 and Au25(pMBA)18 Nano Clusters. J Surgery Emerg Med 1: 21.

75. Alireza Heidari (2017) Developmental Cell Biology in Adult Stem Cells Death and Autophagy to Trigger a Preventive Allergic Reaction to Common Airborne Allergens under Synchrotron Radiation Using Nanotechnology for Therapeutic Goals in Particular Allergy Shots (Immunotherapy). Cell Biol (Henderson, NV) 6: 1.

76. Alireza Heidari (2017) Changing Metal Powder Characteristics for Elimination of the Heavy Metals Toxicity and Diseases in Disruption of Extracellular Matrix (ECM) Proteins Adjustment in Cancer Metastases Induced by Osteosarcoma, Chondrosarcoma, Carcinoid, Carcinoma, Ewing's Sarcoma, Fibrosarcoma and Secondary Hematopoietic Solid or Soft Tissue Tumors. J Powder Metall Min 6: 170.

77. Alireza Heidari (2017) Nanomedicine-Based Combination Anti-Cancer Therapy between Nucleic Acids and Anti-Cancer Nano Drugs in Covalent Nano Drugs Delivery Systems for Selective Imaging and Treatment of Human Brain Tumors Using Hyaluronic Acid, Alguronic Acid and Sodium Hyaluronate as Anti-Cancer Nano Drugs and Nucleic Acids Delivery under Synchrotron Radiation. Am J Drug Deliv 5: 2.

78. Alireza Heidari (2017) Clinical Trials of Dendritic Cell Therapies for Cancer Exposing Vulnerabilities in Human Cancer Cells' Metabolism and Metabolomics: New Discoveries, Unique Features Inform New Therapeutic Opportunities, Biotech's Bumpy Road to the Market and Elucidating the Biochemical Programs that Support Cancer Initiation and Progression. J Biol Med Science 1: e103.

79. Alireza Heidari (2017) The Design Graphene-Based Nanosheets as a New Nanomaterial in Anti-Cancer Therapy and Delivery of Chemotherapeutics and Biological Nano Drugs for Liposomal Anti-Cancer Nano Drugs and Gene Delivery. Br Biomed Bull 5: 305 .

80. Alireza Heidari (2017) Integrative Approach to Biological Networks for Emerging Roles of Proteomics, Genomics and Transcriptomics in the Discovery and Validation of Human Colorectal Cancer Biomarkers from DNA/RNA Sequencing Data under Synchrotron Radiation. Transcriptomics 5: e117.

81. Alireza Heidari (2017) Elimination of the Heavy Metals Toxicity and Diseases in Disruption of Extracellular Matrix (ECM) Proteins and Cell Adhesion Intelligent Nanomolecules Adjustment in Cancer Metastases Using Metalloenzymes and under Synchrotron Radiation. Lett Health Biol Sci 2 (2): 1-4.

82. Alireza Heidari (2017) Treatment of Breast Cancer Brain Metastases through a Targeted Nanomolecule Drug Delivery System Based on Dopamine Functionalized MultiWall Carbon Nanotubes (MWCNTs) Coated with Nano Graphene Oxide (GO) and Protonated Polyaniline (PANI) in Situ During the Polymerization of Aniline Autogenic Nanoparticles for the Delivery of Anti-Cancer Nano Drugs under Synchrotron Radiation. Br J Res, 4 (3): 16.

83. Alireza Heidari (2017) Sedative, Analgesic and Ultrasound-Mediated Gastrointestinal Nano Drugs Delivery for Gastrointestinal Endoscopic Procedure, Nano Drug-Induced Gastrointestinal Disorders and Nano Drug Treatment of Gastric Acidity. Res Rep Gastroenterol, 1: 1 .
84. Alireza Heidari (2017) Synthesis, Pharmacokinetics, Pharmacodynamics, Dosing, Stability, Safety and Efficacy of Orphan Nano Drugs to Treat High Cholesterol and Related Conditions and to Prevent Cardiovascular Disease under Synchrotron Radiation. J Pharm Sci Emerg Drugs 5: 1.

85. Alireza Heidari (2017) Non-Linear Compact Proton Synchrotrons to Improve Human Cancer Cells and Tissues Treatments and Diagnostics through Particle Therapy Accelerators with Monochromatic Microbeams. J Cell Biol Mol Sci 2 (1): 1-5.

86. Alireza Heidari (2017) Design of Targeted Metal Chelation Therapeutics Nanocapsules as Colloidal Carriers and Blood-Brain Barrier (BBB) Translocation to Targeted Deliver Anti-Cancer Nano Drugs into the Human Brain to Treat Alzheimer's Disease under Synchrotron Radiation. J Nanotechnol Material Sci 4 (2): 1-5.

87. Ricardo Gobato, Alireza Heidari (2017) Calculations Using Quantum Chemistry for Inorganic Molecule Simulation BeLi2SeSi. Science Journal of Analytical Chemistry, Vol. 5, No. 6, Pages 76-85.

88. Alireza Heidari (2017) Different High-Resolution Simulations of Medical, Medicinal, Clinical, Pharmaceutical and Therapeutics Oncology of Human Lung Cancer Translational Anti-Cancer Nano Drugs Delivery Treatment Process under Synchrotron and X-Ray Radiations. J Med Oncol. Vol. 1 No. 1: 1.

89. Alireza Heidari (2017) A Modern Ethnomedicinal Technique for Transformation, Prevention and Treatment of Human Malignant Gliomas Tumors into Human Benign Gliomas Tumors under Synchrotron Radiation. Am J Ethnomed, Vol. 4 No. 1: 10.

90. Alireza Heidari (2017) Active Targeted Nanoparticles for Anti-Cancer Nano Drugs Delivery across the Blood-Brain Barrier for Human Brain Cancer Treatment, Multiple Sclerosis (MS) and Alzheimer's Diseases Using Chemical Modifications of AntiCancer Nano Drugs or Drug-Nanoparticles through Zika Virus (ZIKV) Nanocarriers under Synchrotron Radiation. J Med Chem Toxicol, 2 (3): 1-5.

91. Alireza Heidari (2017) Investigation of Medical, Medicinal, Clinical and Pharmaceutical Applications of Estradiol, Mestranol (Norlutin), Norethindrone (NET), Norethisterone Acetate (NETA), Norethisterone Enanthate (NETE) and Testosterone Nanoparticles as Biological Imaging, Cell Labeling, Anti-Microbial Agents and Anti-Cancer Nano Drugs in Nanomedicines Based Drug Delivery Systems for Anti-Cancer Targeting and Treatment. Parana Journal of Science and Education (PJSE)-V.3, n.4, (10-19) October 12.

92. Alireza Heidari (2017) A Comparative Computational and Experimental Study on Different Vibrational Biospectroscopy Methods, Techniques and Applications for Human Cancer Cells in Tumor Tissues Simulation, Modeling, Research, Diagnosis and Treatment. Open J Anal Bioanal Chem 1 (1): 014-020.

93. Alireza Heidari (2017) Combination of DNA/RNA Ligands and Linear/Non-Linear Visible-Synchrotron Radiation-Driven N-Doped Ordered Mesoporous Cadmium Oxide (CdO) Nanoparticles Photocatalysts Channels Resulted in an Interesting Synergistic Effect Enhancing Catalytic Anti-Cancer Activity. Enz Eng 6: 1.

94. Alireza Heidari (2017) Modern Approaches in Designing Ferritin, Ferritin Light Chain, Transferrin, Beta-2 Transferrin and Bacterioferritin-Based Anti-Cancer Nano Drugs Encapsulating Nanosphere as DNA-Binding Proteins from Starved Cells (DPS). Mod Appro Drug Des. 1 (1). MADD.000504.

95. Alireza Heidari (2017) Potency of Human Interferon $\beta-1$ a and Human Interferon $\beta-1 b$ in Enzymotherapy, Immunotherapy, Chemotherapy, Radiotherapy, Hormone Therapy and Targeted Therapy of Encephalomyelitis Disseminate/Multiple Sclerosis (MS) and Hepatitis A, B, C, D, E, F and G Virus Enter and Targets Liver Cells. J Proteomics Enzymol 6: 1 .

96. Alireza Heidari (2017) Transport Therapeutic Active Targeting of Human Brain Tumors Enable Anti-Cancer Nanodrugs Delivery across the Blood-Brain Barrier (BBB) to Treat Brain Diseases Using Nanoparticles and Nanocarriers under Synchrotron Radiation. J Pharm Pharmaceutics 4 (2): 1-5.

97. Alireza Heidari, Christopher Brown (2017) Combinatorial Therapeutic Approaches to DNA/RNA and Benzylpenicillin (Penicillin G), Fluoxetine Hydrochloride (Prozac and Sarafem), Propofol (Diprivan), Acetylsalicylic Acid (ASA) (Aspirin), Naproxen Sodium (Aleve and Naprosyn) and Dextromethamphetamine Nanocapsules with Surface Conjugated DNA/RNA to Targeted Nano Drugs for Enhanced Anti-Cancer Efficacy and Targeted Cancer Therapy Using Nano Drugs Delivery Systems. Ann Adv Chem. 1 (2): 061-069.

98. Alireza Heidari (2017) High-Resolution Simulations of Human Brain Cancer Translational Nano Drugs Delivery Treatment Process under Synchrotron Radiation. J Transl Res. 1 (1): 1-3.

99. Alireza Heidari (2017) Investigation of Anti-Cancer Nano Drugs' Effects' Trend on Human Pancreas Cancer Cells and Tissues Prevention, Diagnosis and Treatment Process under Synchrotron and X-Ray Radiations with the Passage of Time Using Mathematica. Current Trends Anal Bioanal Chem, 1 (1): 36-41. 
100. Alireza Heidari (2017) Pros and Cons Controversy on Molecular Imaging and Dynamics of Double-Standard DNA/RNA of Human Preserving Stem CellsBinding Nano Molecules with Androgens/Anabolic Steroids (AAS) or Testosterone Derivatives through Tracking of Helium-4 Nucleus (Alpha Particle) Using Synchrotron Radiation. Arch Biotechnol Biomed. 1 (1): 067-0100.

101. Alireza Heidari (2017) Visualizing Metabolic Changes in Probing Human Cancer Cells and Tissues Metabolism Using Vivo 1H or Proton NMR, 13C NMR, 15N NMR and 31P NMR Spectroscopy and Self-Organizing Maps under Synchrotron Radiation. SOJ Mater Sci Eng 5 (2): 1-6.

102. Alireza Heidari (2017) Cavity Ring-Down Spectroscopy (CRDS), Circular Dichroism Spectroscopy, Cold Vapour Atomic Fluorescence Spectroscopy and Correlation Spectroscopy Comparative Study on Malignant and Benign Human Cancer Cells and Tissues with the Passage of Time under Synchrotron Radiation. Enliven: Challenges Cancer Detect Ther 4 (2): e001.

103. Alireza Heidari (2017) Laser Spectroscopy, Laser-Induced Breakdown Spectroscopy and Laser-Induced Plasma Spectroscopy Comparative Study on Malignant and Benign Human Cancer Cells and Tissues with the Passage of Time under Synchrotron Radiation. Int J Hepatol Gastroenterol, 3 (4): 079-084.

104. Alireza Heidari (2017) Time-Resolved Spectroscopy and Time-Stretch Spectroscopy Comparative Study on Malignant and Benign Human Cancer Cells and Tissues with the Passage of Time under Synchrotron Radiation. Enliven: Pharmacovigilance and Drug Safety 4 (2): e001.

105. Alireza Heidari (2017) Overview of the Role of Vitamins in Reducing Negative Effect of Decapeptyl (Triptorelin Acetate or Pamoate Salts) on Prostate Cancer Cells and Tissues in Prostate Cancer Treatment Process through Transformation of Malignant Prostate Tumors into Benign Prostate Tumors under Synchrotron Radiation. Open J Anal Bioanal Chem 1 (1): 021-026.

106. Alireza Heidari (2017) Electron Phenomenological Spectroscopy, Electron Paramagnetic Resonance (EPR) Spectroscopy and Electron Spin Resonance (ESR) Spectroscopy Comparative Study on Malignant and Benign Human Cancer Cells and Tissues with the Passage of Time under Synchrotron Radiation. Austin J Anal Pharm Chem. 4 (3): 1091.

107. Alireza Heidari (2017) Therapeutic Nanomedicine Different High-Resolution Experimental Images and Computational Simulations for Human Brain Cancer Cells and Tissues Using Nanocarriers Deliver DNA/RNA to Brain Tumors under Synchrotron Radiation with the Passage of Time Using Mathematica and MATLAB. Madridge J Nano Tech. Sci. 2 (2): 77-83.

108. Alireza Heidari (2017) A Consensus and Prospective Study on Restoring Cadmium Oxide (CdO) Nanoparticles Sensitivity in Recurrent Ovarian Cancer by Extending the Cadmium Oxide (CdO) Nanoparticles-Free Interval Using Synchrotron Radiation Therapy as Antibody-Drug Conjugate for the Treatment of Limited-Stage Small Cell Diverse Epithelial Cancers. Cancer Clin Res Rep, 1: 2, e001.

109. Alireza Heidari (2017) A Novel and Modern Experimental Imaging and Spectroscopy Comparative Study on Malignant and Benign Human Cancer Cells and Tissues with the Passage of Time under White Synchrotron Radiation. Cancer Sci Res Open Access 4 (2): 1-8.

110. Alireza Heidari (2017) Different High-Resolution Simulations of Medical, Medicinal, Clinical, Pharmaceutical and Therapeutics Oncology of Human Breast Cancer Translational Nano Drugs Delivery Treatment Process under Synchrotron and X-Ray Radiations. J Oral Cancer Res 1 (1): 12-17.

111. Alireza Heidari (2017) Vibrational Decihertz (dHz), Centihertz (cHz), Millihertz $(\mathrm{mHz})$, Microhertz $(\mu \mathrm{Hz})$, Nanohertz $(\mathrm{nHz})$, Picohertz $(\mathrm{pHz})$, Femtohertz $(\mathrm{fHz})$, Attohertz (aHz), Zeptohertz (zHz) and Yoctohertz (yHz) Imaging and Spectroscopy Comparative Study on Malignant and Benign Human Cancer Cells and Tissues under Synchrotron Radiation. International Journal of Biomedicine, 7 (4), 335-340.

112. Alireza Heidari (2017) Force Spectroscopy and Fluorescence Spectroscopy Comparative Study on Malignant and Benign Human Cancer Cells and Tissues with the Passage of Time under Synchrotron Radiation. EC Cancer, 2 (5), 239-246.

113. Alireza Heidari (2017) Photoacoustic Spectroscopy, Photoemission Spectroscopy and Photothermal Spectroscopy Comparative Study on Malignant and Benign Human Cancer Cells and Tissues with the Passage of Time under Synchrotron Radiation. BAOJ Cancer Res Ther, 3: 3, 045-052.

114. Alireza Heidari (2017) J-Spectroscopy, Exchange Spectroscopy (EXSY), Nucle $\neg$ ar Overhauser Effect Spectroscopy (NOESY) and Total Correlation Spectroscopy (TOCSY) Comparative Study on Malignant and Benign Human Cancer Cells and Tissues under Synchrotron Radiation. EMS Eng Sci J, 1 (2): 006-013.

115. Alireza Heidari (2017) Neutron Spin Echo Spectroscopy and Spin Noise Spectroscopy Comparative Study on Malignant and Benign Human Cancer Cells and Tissues with the Passage of Time under Synchrotron Radiation. Int J Biopharm Sci, 1: 103-107.
116. Alireza Heidari (2017) Vibrational Decahertz (daHz), Hectohertz (hHz), Kilohertz $(\mathrm{kHz})$, Megahertz $(\mathrm{MHz})$, Gigahertz $(\mathrm{GHz})$, Terahertz $(\mathrm{THz})$, Petahertz (PHz), Exahertz $(\mathrm{EHz})$, Zettahertz $(\mathrm{ZHz})$ and Yottahertz $(\mathrm{YHz})$ Imaging and Spectroscopy Comparative Study on Malignant and Benign Human Cancer Cells and Tissues under Synchrotron Radiation. Madridge J Anal Sci Instrum, 2 (1): 41-46.

117. Alireza Heidari (2018) Two-Dimensional Infrared Correlation Spectroscopy, Linear Two-Dimensional Infrared Spectroscopy and Non-Linear Two-Dimensional Infrared Spectroscopy Comparative Study on Malignant and Benign Human Cancer Cells and Tissues under Synchrotron Radiation with the Passage of Time. J Mater Sci Nanotechnol 6 (1): 101

118. Alireza Heidari (2018) Fourier Transform Infrared (FTIR) Spectroscopy, NearInfrared Spectroscopy (NIRS) and Mid-Infrared Spectroscopy (MIRS) Comparative Study on Malignant and Benign Human Cancer Cells and Tissues under Synchrotron Radiation with the Passage of Time. Int J Nanotechnol Nanomed, Volume 3, Issue 1, Pages 1-6.

119. Alireza Heidari (2018) Infrared Photo Dissociation Spectroscopy and Infrared Correlation Table Spectroscopy Comparative Study on Malignant and Benign Human Cancer Cells and Tissues under Synchrotron Radiation with the Passage of Time. Austin Pharmacol Pharm, 3 (1): 1011.

120. Alireza Heidari (2018) Novel and Transcendental Prevention, Diagnosis and Treatment Strategies for Investigation of Interaction among Human Blood Cancer Cells, Tissues, Tumors and Metastases with Synchrotron Radiation under AntiCancer Nano Drugs Delivery Efficacy Using MATLAB Modeling and Simulation. Madridge J Nov Drug Res, 1 (1): 18-24.

121. Alireza Heidari (2018) Comparative Study on Malignant and Benign Human Cancer Cells and Tissues with the Passage of Time under Synchrotron Radiation. Open Access J Trans Med Res, 2 (1): 00026-00032.

122. Marcia Regina Risso Gobato, Ricardo Gobato, Alireza Heidari (2018) Planting of Jaboticaba Trees for Landscape Repair of Degraded Area. Landscape Architecture and Regional Planning, Vol. 3, No. 1, 2018, Pages 1-9.

123. Alireza Heidari (2018) Fluorescence Spectroscopy, Phosphorescence Spectroscopy and Luminescence Spectroscopy Comparative Study on Malignant and Benign Human Cancer Cells and Tissues under Synchrotron Radiation with the Passage of Time. SM J Clin. Med. Imaging, 4 (1): 1018.

124. Alireza Heidari (2018) Nuclear Inelastic Scattering Spectroscopy (NISS) and Nuclear Inelastic Absorption Spectroscopy (NIAS) Comparative Study on Malignant and Benign Human Cancer Cells and Tissues under Synchrotron Radiation. Int J Pharm Sci, 2 (1): 1-14.

125. Alireza Heidari (2018) X-Ray Diffraction (XRD), Powder X-Ray Diffraction (PXRD) and Energy-Dispersive X-Ray Diffraction (EDXRD) Comparative Study on Malignant and Benign Human Cancer Cells and Tissues under Synchrotron Radiation. J Oncol Res; 2 (1): 1-14.

126. Alireza Heidari (2018) Correlation Two-Dimensional Nuclear Magnetic Reso $\neg$ nance (NMR) (2D-NMR) (COSY) Imaging and Spectrosco $\neg$ py Comparative Study on Malignant and Benign Human Cancer Cells and Tissues under Synchrotron Radiation. EMS Can Sci, 1-1-001.

127. Alireza Heidari (2018) Thermal Spectroscopy, Photothermal Spectroscopy, Therma Microspectroscopy, Photothermal Microspectroscopy, Thermal Macrospectroscopy and Photothermal Macrospectroscopy Comparative Study on Malignant and Benign Human Cancer Cells and Tissues with the Passage of Time under Synchrotron Radiation. SM J Biometrics Biostat, 3 (1): 1024.

128. Alireza Heidari (2018) A Modern and Comprehensive Experimental Biospectroscopic Comparative Study on Human Common Cancers' Cells, Tissues and Tumors before and after Synchrotron Radiation Therapy. Open Acc J Oncol Med. 1 (1).

129. Alireza Heidari (2018) Heteronuclear Correlation Experiments such as Heteronuclear Single-Quantum Correlation Spectroscopy (HSQC), Heteronuclear MultipleQuantum Correlation Spectroscopy (HMQC) and Heteronuclear Multiple-Bond Correlation Spectroscopy (HMBC) Comparative Study on Malignant and Benign Human Endocrinology and Thyroid Cancer Cells and Tissues under Synchrotron Radiation. J Endocrinol Thyroid Res, 3 (1): 555603.

130. Alireza Heidari (2018) Nuclear Resonance Vibrational Spectroscopy (NRVS), Nuclear Inelastic Scattering Spectroscopy (NISS), Nuclear Inelastic Absorption Spectroscopy (NIAS) and Nuclear Resonant Inelastic X-Ray Scattering Spectroscopy (NRIXSS) Comparative Study on Malignant and Benign Human Cancer Cells and Tissues under Synchrotron Radiation. Int J Bioorg Chem Mol Biol. 6 (1e): 1-5.

131. Alireza Heidari (2018) A Novel and Modern Experimental Approach to Vibrational Circular Dichroism Spectroscopy and Video Spectroscopy Comparative Study on Malignant and Benign Human Cancer Cells and Tissues with the Passage of Time under White and Monochromatic Synchrotron Radiation. Glob J Endocrinol Metab. 1 (3). GJEM. 000514-000519. 
132. Alireza Heidari (2018) Pros and Cons Controversy on Heteronuclear Correlation Experiments such as Heteronuclear Single-Quantum Correlation Spectroscopy (HSQC), Heteronuclear Multiple-Quantum Correlation Spectroscopy (HMQC) and Heteronuclear Multiple-Bond Correlation Spectroscopy (HMBC) Comparative Study on Malignant and Benign Human Cancer Cells and Tissues under Synchrotron Radiation. EMS Pharma J. 1 (1): 002.

133. Alireza Heidari (2018) A Modern Comparative and Comprehensive Experimental Biospectroscopic Study on Different Types of Infrared Spectroscopy of Malignant and Benign Human Cancer Cells and Tissues with the Passage of Time under Synchrotron Radiation. J Analyt Molecul Tech. 3 (1): 8.

134. Alireza Heidari (2018) Investigation of Cancer Types Using Synchrotron Technology for Proton Beam Therapy: An Experimental Biospectroscopic Comparative Study. European Modern Studies Journal, Vol. 2, No. 1, 13-29.

135. Alireza Heidari (2018) Saturated Spectroscopy and Unsaturated Spectroscopy Comparative Study on Malignant and Benign Human Cancer Cells and Tissues with the Passage of Time under Synchrotron Radiation. Imaging J Clin Medical Sci. 5 (1): 001-007.

136. Alireza Heidari (2018) Small-Angle Neutron Scattering (SANS) and Wide-Angle X-Ray Diffraction (WAXD) Comparative Study on Malignant and Benign Human Cancer Cells and Tissues under Synchrotron Radiation. Int J Bioorg Chem Mol Biol. $6(2 \mathrm{e}): 1-6$.

137. Alireza Heidari (2018) Investigation of Bladder Cancer, Breast Cancer, Colorectal Cancer, Endometrial Cancer, Kidney Cancer, Leukemia, Liver, Lung Cancer, Melanoma, Non-Hodgkin Lymphoma, Pancreatic Cancer, Prostate Cancer, Thyroid Cancer and Non-Melanoma Skin Cancer Using Synchrotron Technology for Proton Beam Therapy: An Experimental Biospectroscopic Comparative Study. Ther Res Skin Dis 1 (1)

138. Alireza Heidari (2018) Attenuated Total Reflectance Fourier Transform Infrared (ATR-FTIR) Spectroscopy, Micro-Attenuated Total Reflectance Fourier Transform Infrared (Micro-ATR-FTIR) Spectroscopy and Macro-Attenuated Total Reflectance Fourier Transform Infrared (Macro-ATR-FTIR) Spectroscopy Comparative Study on Malignant and Benign Human Cancer Cells and Tissues under Synchrotron Radiation with the Passage of Time. International Journal of Chemistry Papers, 2 (1): $1-12$.

139. Alireza Heidari (2018) Mössbauer Spectroscopy, Mössbauer Emission Spectroscopy and 57Fe Mössbauer Spectroscopy Comparative Study on Malignant and Benign Human Cancer Cells and Tissues under Synchrotron Radiation. Acta Scientific Cancer Biology 2.3: 17-20.

140. Alireza Heidari (2018) Comparative Study on Malignant and Benign Human Cancer Cells and Tissues under Synchrotron Radiation with the Passage of Time. Organic \& Medicinal Chem IJ. 6 (1): 555676.

141. Alireza Heidari (2018) Correlation Spectroscopy, Exclusive Correlation Spectroscopy and Total Correlation Spectroscopy Comparative Study on Malignant and Benign Human AIDS-Related Cancers Cells and Tissues with the Passage of Time under Synchrotron Radiation. Int J Bioanal Biomed. 2 (1): 001-007.

142. Alireza Heidari (2018) Biomedical Instrumentation and Applications of Biospectroscopic Methods and Techniques in Malignant and Benign Human Cancer Cells and Tissues Studies under Synchrotron Radiation and Anti-Cancer Nano Drugs Delivery. Am J Nanotechnol Nanomed. 1 (1): 001-009.

143. Alireza Heidari (2018) Vivo 1H or Proton NMR, 13C NMR, 15N NMR and 31P NMR Spectroscopy Comparative Study on Malignant and Benign Human Cancer Cells and Tissues under Synchrotron Radiation. Ann Biomet Biostat. 1 (1): 1001.

144. Alireza Heidari (2018) Grazing-Incidence Small-Angle Neutron Scattering (GISANS) and Grazing-Incidence X-Ray Diffraction (GIXD) Comparative Study on Malignant and Benign Human Cancer Cells, Tissues and Tumors under Synchrotron Radiation. Ann Cardiovasc Surg. 1 (2): 1006.

145. Alireza Heidari (2018) Adsorption Isotherms and Kinetics of Multi-Walled Carbon Nanotubes (MWCNTs), Boron Nitride Nanotubes (BNNTs), Amorphous Boron Nitride Nanotubes (a-BNNTs) and Hexagonal Boron Nitride Nanotubes (h-BNNTs) for Eliminating Carcinoma, Sarcoma, Lymphoma, Leukemia, Germ Cell Tumor and Blastoma Cancer Cells and Tissues. Clin Med Rev Case Rep 5: 201.

146. Alireza Heidari (2018) Correlation Spectroscopy (COSY), Exclusive Correlation Spectroscopy (ECOSY), Total Correlation Spectroscopy (TOCSY), Incredible Natural-Abundance Double-Quantum Transfer Experiment (INADEQUATE), Heteronuclear Single-Quantum Correlation Spectroscopy (HSQC), Heteronuclear Multiple-Bond Correlation Spectroscopy (HMBC), Nuclear Overhauser Effect Spectroscopy (NOESY) and Rotating Frame Nuclear Overhauser Effect Spectroscopy (ROESY) Comparative Study on Malignant and Benign Human Cancer Cells and Tissues under Synchrotron Radiation. Acta Scientific Pharmaceutical Sciences 2.5: $30-35$.
147. Alireza Heidari (2018) Small-Angle X-Ray Scattering (SAXS), Ultra-Small Angle X-Ray Scattering (USAXS), Fluctuation X-Ray Scattering (FXS), Wide-Angle X-Ray Scattering (WAXS), Grazing-Incidence Small-Angle X-Ray Scattering (GISAXS), Grazing-Incidence Wide-Angle X-Ray Scattering (GIWAXS), SmallAngle Neutron Scattering (SANS), Grazing-Incidence Small-Angle Neutron Scattering (GISANS), X-Ray Diffraction (XRD), Powder X-Ray Diffraction (PXRD), Wide-Angle X-Ray Diffraction (WAXD), Grazing-Incidence XRay Diffraction (GIXD) and Energy-Dispersive X-Ray Diffraction (EDXRD) Comparative Study on Malignant and Benign Human Cancer Cells and Tissues under Synchrotron Radiation. Oncol Res Rev, Volume 1 (1): 1-10.

148. Alireza Heidari (2018) Pump-Probe Spectroscopy and Transient Grating Spectroscopy Comparative Study on Malignant and Benign Human Cancer Cells and Tissues with the Passage of Time under Synchrotron Radiation. Adv Material Sci Engg, Volume 2, Issue 1, Pages 1-7.

149. Alireza Heidari (2018) Grazing-Incidence Small-Angle X-Ray Scattering (GISAXS) and Grazing-Incidence Wide-Angle X-Ray Scattering (GIWAXS) Comparative Study on Malignant and Benign Human Cancer Cells and Tissues under Synchrotron Radiation. Insights Pharmacol Pharm Sci 1 (1): 1-8.

150. Alireza Heidari (2018) Acoustic Spectroscopy, Acoustic Resonance Spectroscopy and Auger Spectroscopy Comparative Study on Anti-Cancer Nano Drugs Delivery in Malignant and Benign Human Cancer Cells and Tissues with the Passage of Time under Synchrotron Radiation. Nanosci Technol 5 (1): 1-9.

151. Alireza Heidari (2018) Niobium, Technetium, Ruthenium, Rhodium, Hafnium, Rhenium, Osmium and Iridium Ions Incorporation into the Nano Polymeric Matrix (NPM) by Immersion of the Nano Polymeric Modified Electrode (NPME) as Molecular Enzymes and Drug Targets for Human Cancer Cells, Tissues and Tumors Treatment under Synchrotron and Synchrocyclotron Radiations. Nanomed Nanotechnol, 3 (2): 000138.

152. Alireza Heidari (2018) Homonuclear Correlation Experiments such as Homonuclear Single- Quantum Correlation Spectroscopy (HSQC), Homonuclear MultipleQuantum Correlation Spectroscopy (HMQC) and Homonuclear Multiple-Bond Correlation Spectroscopy (HMBC) Comparative Study on Malignant and Benign Human Cancer Cells and Tissues under Synchrotron Radiation. Austin J Proteomics Bioinform \& Genomics. 5 (1): 1024.

153. Alireza Heidari (2018) Atomic Force Microscopy Based Infrared (AFM-IR) Spectroscopy and Nuclear Resonance Vibrational Spectroscopy Comparative Study on Malignant and Benign Human Cancer Cells and Tissues under Synchrotron Radiation with the Passage of Time. J Appl Biotechnol Bioeng. 5 (3): 142?148.

154. Alireza Heidari (2018) Time-Dependent Vibrational Spectral Analysis of Malignant and Benign Human Cancer Cells and Tissues under Synchrotron Radiation. J Cancer Oncol, 2 (2): 000124

155. Alireza Heidari (2018) Palauamine and Olympiadane Nano Molecules Incorporation into the Nano Polymeric Matrix (NPM) by Immersion of the Nano Polymeric Modified Electrode (NPME) as Molecular Enzymes and Drug Targets for Human Cancer Cells, Tissues and Tumors Treatment under Synchrotron and Synchrocyclotron Radiations. Arc Org Inorg Chem Sci 3(1).

156. Ricardo Gobato, Alireza Heidari (2018) Infrared Spectrum and Sites of Action of Sanguinarine by Molecular Mechanics and ab initio Methods. International Journal of Atmospheric and Oceanic Sciences. Vol. 2, No. 1, pp. 1-9.

157. Alireza Heidari (2018) Gamma Linolenic Methyl Ester, 5-Heptadeca-5,8,11 Trienyl 1,3,4-Oxadiazole-2-Thiol, Sulphoquinovosyl Diacyl Glycerol, Ruscogenin, Nocturnoside B, Protodioscine B, Parquisoside-B, Leiocarposide, Narangenin, 7Methoxy Hespertin, Lupeol, Rosemariquinone, Rosmanol and Rosemadiol Nano Molecules Incorporation into the Nano Polymeric Matrix (NPM) by Immersion of the Nano Polymeric Modifi ed Electrode (NPME) as Molecular Enzymes and Drug Targets for Human Cancer Cells, Tissues and Tumors Treatment under Synchrotron and Synchrocyclotron Radiations. Int J Pharma Anal Acta. 2 (1): 007-014.

158. Alireza Heidari (2018) Angelic Acid, Diabolic Acids, Draculin and Miraculin Nano Molecules Incorporation into the Nano Polymeric Matrix (NPM) by Immersion of the Nano Polymeric Modified Electrode (NPME) as Molecular Enzymes and Drug Targets for Human Cancer Cells, Tissues and Tumors Treatment Under Synchrotron and Synchrocyclotron Radiations. Med \& Analy Chem Int J, 2 (1): 000111.

159. Alireza Heidari (2018) Fourier Transform Infrared (FTIR) Spectroscopy, Attenuated Total Reflectance Fourier Transform Infrared (ATR-FTIR) Spectroscopy, MicroAttenuated Total Reflectance Fourier Transform Infrared (Micro-ATR-FTIR) Spectroscopy, Macro- Attenuated Total Reflectance Fourier Transform Infrared (Macro-ATR-FTIR) Spectroscopy, Two-Dimensional Infrared Correlation Spectroscopy, Linear Two-Dimensional Infrared Spectroscopy, Non-Linear TwoDimensional Infrared Spectroscopy, Atomic Force Microscopy Based Infrared (AFM-IR) Spectroscopy, Infrared Photodissociation Spectroscopy, Infrared 
Correlation Table Spectroscopy, Near-Infrared Spectroscopy (NIRS), Mid-Infrared Spectroscopy (MIRS), Nuclear Resonance Vibrational Spectroscopy, Thermal Infrared Spectroscopy and Photothermal Infrared Spectroscopy Comparative Study on Malignant and Benign Human Cancer Cells and Tissues under Synchrotron Radiation with the Passage of Time. Glob Imaging Insights, Volume 3 (2): 1-14

160. Alireza Heidari (2018) Tetrakis [3, 5-bis (Trifluoromethyl) Phenyl] Borate (BARF)Enhanced Precatalyst Preparation Stabilization and Initiation (EPPSI) Nano Molecules. Medical Research and Clinical Case Reports 2.1: 112-125.

161. Alireza Heidari (2018) Heteronuclear Single-Quantum Correlation Spectroscopy (HSQC) and Heteronuclear Multiple-Bond Correlation Spectroscopy (HMBC) Comparative Study on Malignant and Benign Human Cancer Cells, Tissues and Tumors under Synchrotron and Synchrocyclotron Radiations. Chronicle of Medicine and Surgery 2.3 : $151-163$.

162. Alireza Heidari (2018) Sydnone, Münchnone, Montréalone, Mogone, Montelukast, Quebecol and Palau'amine-Enhanced Precatalyst Preparation Stabilization and Initiation (EPPSI) Nano Molecules. Sur Cas Stud Op Acc J. 1 (3)

163. Alireza Heidari (2018) Fornacite, Orotic Acid, Rhamnetin, Sodium Ethyl Xanthate (SEX) and Spermine (Spermidine or Polyamine) Nanomolecules Incorporation into the Nanopolymeric Matrix (NPM). International Journal of Biochemistry and Biomolecules, Vol. 4: Issue 1: 1-19.

164. Ricardo Gobato, Alireza Heidari (2018) Molecular Mechanics and Quantum Chemical Study on Sites of Action of Sanguinarine Using Vibrational Spectroscopy Based on Molecular Mechanics and Quantum Chemical Calculations. Malaysian Journal of Chemistry, Vol. 20(1), 1-23.

165. Alireza Heidari (2018) Vibrational Spectroscopic Studies on Anti-cancer Nanopharmaceuticals (Part I). Malaysian Journal of Chemistry, Vol. 20(1), 33 - 73.

166. Alireza Heidari (2018) Vibrational Spectroscopic Studies on Anti-cancer Nanopharmaceuticals (Part II). Malaysian Journal of Chemistry, Vol. 20(1), 74 - 117.

167. Alireza Heidari, Ricardo Gobato (2018) Putrescine, Cadaverine, Spermine and Spermidine- Enhanced Precatalyst Preparation Stabilization and Initiation (EPPSI) Nano Molecules. Parana Journal of Science and Education (PJSE)-v.4, n.5, (1-14).

168. Alireza Heidari (2018) Cadaverine (1,5-Pentanediamine or Pentamethylenediamine), Diethyl Azodicarboxylate (DEAD or DEADCAT) and Putrescine (Tetramethylenediamine) Nano Molecules Incorporation into the Nano Polymeric Matrix (NPM) by Immersion of the Nano Polymeric Modified Electrode (NPME) as Molecular Enzymes and Drug Targets for Human Cancer Cells, Tissues and Tumors Treatment under Synchrotron and Synchrocyclotron Radiations. Hiv and Sexual Health Open Access Open Journal. 1 (1): 4-11.

169. Alireza Heidari (2018) Uranocene (U(C8H8)2) and Bis(Cyclooctatetraene)Iron $(\mathrm{Fe}(\mathrm{C} 8 \mathrm{H} 8) 2$ or $\mathrm{Fe}(\mathrm{COT}) 2)$-Enhanced Precatalyst Preparation Stabilization and Initiation (EPPSI) Nano Molecules. Chemistry Reports. 1 (2): 1-16.

170. Alireza Heidari (2018) Improving the Performance of Nano-Endofullerenes in Polyaniline Nanostructure-Based Biosensors by Covering Californium Colloidal Nanoparticles with Multi-Walled Carbon Nanotubes. Journal of Advances in Nanomaterials, Vol. 3, No. 1, Pages 1-28.

171. Alireza Heidari (2018) Biomedical Systematic and Emerging Technological Study on Human Malignant and Benign Cancer Cells and Tissues Biospectroscopic Analysis under Synchrotron Radiation. Glob Imaging Insights, Volume 3 (3): 1-7.

172. Alireza Heidari (2018) Deep-Level Transient Spectroscopy and X-Ray Photoelectron Spectroscopy (XPS) Comparative Study on Malignant and Benign Human Cancer Cells and Tissues with the Passage of Time under Synchrotron Radiation. Res Dev Material Sci. 7 (2). RDMS.000659.
173. Alireza Heidari (2018) C70-Carboxyfullerenes Nano Molecules Incorporation into the Nano Polymeric Matrix (NPM) by Immersion of the Nano Polymeric Modified Electrode (NPME) as Molecular Enzymes and Drug Targets for Human Cancer Cells, Tissues and Tumors Treatment under Synchrotron and Synchrocyclotron Radiations. Glob Imaging Insights, Volume 3 (3): 1-7.

174. Alireza Heidari (2018) The Effect of Temperature on Cadmium Oxide (CdO) Nanoparticles Produced by Synchrotron Radiation in the Human Cancer Cells, Tissues and Tumors. International Journal of Advanced Chemistry, 6 (2) 140-156.

175. Alireza Heidari (2018) A Clinical and Molecular Pathology Investigation of Correlation Spectroscopy (COSY), Exclusive Correlation Spectroscopy (ECOSY), Total Correlation Spectroscopy (TOCSY), Heteronuclear Single-Quantum Correlation Spectroscopy (HSQC) and Heteronuclear Multiple-Bond Correlation Spectroscopy (HMBC) Comparative Study on Malignant and Benign Human Cancer Cells, Tissues and Tumors under Synchrotron and Synchrocyclotron Radiations Using Cyclotron versus Synchrotron, Synchrocyclotron and the Large Hadron Collider (LHC) for Delivery of Proton and Helium Ion (Charged Particle) Beams for Oncology Radiotherapy. European Journal of Advances in Engineering and Technology, 5 (7): 414-426.

176. Alireza Heidari (2018) Nano Molecules Incorporation into the Nano Polymeric Matrix (NPM) by Immersion of the Nano Polymeric Modified Electrode (NPME) as Molecular Enzymes and Drug Targets for Human Cancer Cells, Tissues and Tumors Treatment under Synchrotron and Synchrocyclotron Radiations. J Oncol Res. 1 (1): $1-20$.

177. Alireza Heidari (2018) Use of Molecular Enzymes in the Treatment of Chronic Disorders. Canc Oncol Open Access J. 1 (1): 12-15.

178. Alireza Heidari (2018) Vibrational Biospectroscopic Study and Chemical Structure Analysis of Unsaturated Polyamides Nanoparticles as Anti-Cancer Polymeric Nanomedicines Using Synchrotron Radiation. International Journal of Advanced Chemistry, 6 (2) 167-189.

179. Alireza Heidari (2018) Adamantane, Irene, Naftazone and Pyridine-Enhanced Precatalyst Preparation Stabilization and Initiation (PEPPSI) Nano Molecules. Madridge J Nov Drug Res. 2 (1): 61-67.

180. Alireza Heidari (2018) Heteronuclear Single-Quantum Correlation Spectroscopy (HSQC) and Heteronuclear Multiple-Bond Correlation Spectroscopy (HMBC) Comparative Study on Malignant and Benign Human Cancer Cells and Tissues with the Passage of Time under Synchrotron Radiation. Madridge J Nov Drug Res. 2 (1): 68-74.

181. Alireza Heidari, Ricardo Gobato (2018) A Novel Approach to Reduce Toxicities and to Improve Bioavailabilities of DNA/RNA of Human Cancer Cells-Containing Cocaine (Coke), Lysergide (Lysergic Acid Diethyl Amide or LSD), ??-Tetrahydrocannabinol (THC) [(-)-trans-??-Tetrahydrocannabinol], Theobromine (Xantheose), Caffeine, Aspartame (APM) (NutraSweet) and Zidovudine (ZDV) [Azidothymidine (AZT)] as Anti-Cancer Nano Drugs by Coassembly of Dual Anti-Cancer Nano Drugs to Inhibit DNA/RNA of Human Cancer Cells Drug Resistance. Parana Journal of Science and Education, v. 4, n. 5, pp. 1-14.

182. Alireza Heidari, Ricardo Gobato (2018) Ultraviolet Photoelectron Spectroscopy (UPS) and Ultraviolet-Visible (UV-Vis) Spectroscopy Comparative Study on Malignant and Benign Human Cancer Cells and Tissues with the Passage of Time under Synchrotron Radiation. Parana Journal of Science and Education, v. 4, n. 6, pp. $1-14$.

Copyright: (C2018 Heidari A. This is an open-access article distributed under the terms of the Creative Commons Attribution License, which permits unrestricted use, distribution, and reproduction in any medium, provided the original author and source are credited. 